Umweltzeichen für Warmwasserspeicher

\section{Nachwuchs für den Blauen Engel}

Bislang gab es kein Umweltzeichen für Warmwasserspeicher, trotz der hohen energetischen Relevanz dieser Komponente. Jetzt wurde eine Vergabegrundlage erarbeitet, welche die Jury Umweltzeichen am 16. Mai verabschiedet hat. Von Florian Wetzig

D as Umweltzeichen Blauer Engel ist ein Informationsinstrument zur Kommunikation umwelt- und gesundheitsrelevanter Eigenschaften eines Produktes. Ziel der Kennzeichnung ist die Förderung umweltfreundlicher Produkte. Der Blaue Engel gibt den Verbrauchern Orientierung bei der Suche nach einem umweltgerechten Produkt und unterstützt einen nachhaltigen Konsum.

Der Entwicklung eines Kriterienkatalogs für ein Umweltzeichen Blauer Engel geht in der Regel eine Analyse der umweltrelevanten Eigenschaften der betroffenen Produkte voraus. Darauf basierend werden Kriterien formuliert, die eine Kennzeichnung der ökologisch vorteilhaften Produkte erlauben. Anschließend wird der Kriterienkatalog mit Interessengruppen wie den Branchenvertretern diskutiert. Durch diese Beteiligungsmöglichkeit können oft eine höhere Akzeptanz des Blauen Engels bei den Unternehmen erreicht und Zeichennehmer gewonnen werden. Allerdings setzt dies konstruktive Vorschläge und ein produktives Mitarbeiten der beteiligten Akteure voraus. Die abgestimmte Vergabegrundlage wird schließlich der Jury Umweltzeichen zum Beschluss vorgelegt (1).

Ein Schwerpunkt des Blauen Engels liegt bei Produkten zur Wärmeerzeugung in Gebäuden. So ist der Blaue Engel schon viele Jahre für zahlreiche Komponenten der Wärmeerzeugung ein Wegweiser zu umweltfreundlichen und qualitativ hoch- wertigen Produkten. Gleichzeitig konnte durch die eingetretene Marktdifferenzierung eine Verbesserung der Umwelteigenschaften der am Markt vertretenen Produkte erreicht werden. Für Warmwasserspeicher gab es bisher jedoch keine Kennzeichnung. Dabei stellen Warmwasserspeicher eine wichtige Komponente des Wärmeerzeugungssystems dar, insbesondere aufgrund des zunehmenden Einsatzes regenerativer Energieträger in vielen Haushalten.

\section{Puffer-, Kombi- und Trink- wasserspeicher}

Grundsätzlich lassen sich Warmwasserspeicher in die Produktgruppen Puffer-, Kombi-, und Trinkwasserspeicher untergliedern. Als Pufferspeicher werden solche Geräte bezeichnet, die ausschließlich Heizwärme speichern, während Kombispeicher neben Heizwärme auch heißes Trinkwasser zur Verfügung stellen. Trinkwasserspeicher halten kontinuierlich heißes Trinkwasser bereit.

Alle drei Speichertypen gibt es als monovalente und bivalente Speicher. Bei monovalenten Speichern findet die Wärmebereitstellung in der Regel durch einen steuerbaren Energieträger statt, entweder direkt über einen eingebauten Gas- oder Ölbrenner oder indirekt über den Gas-, Öl- oder Pelletbrenner der Heizungsanla-

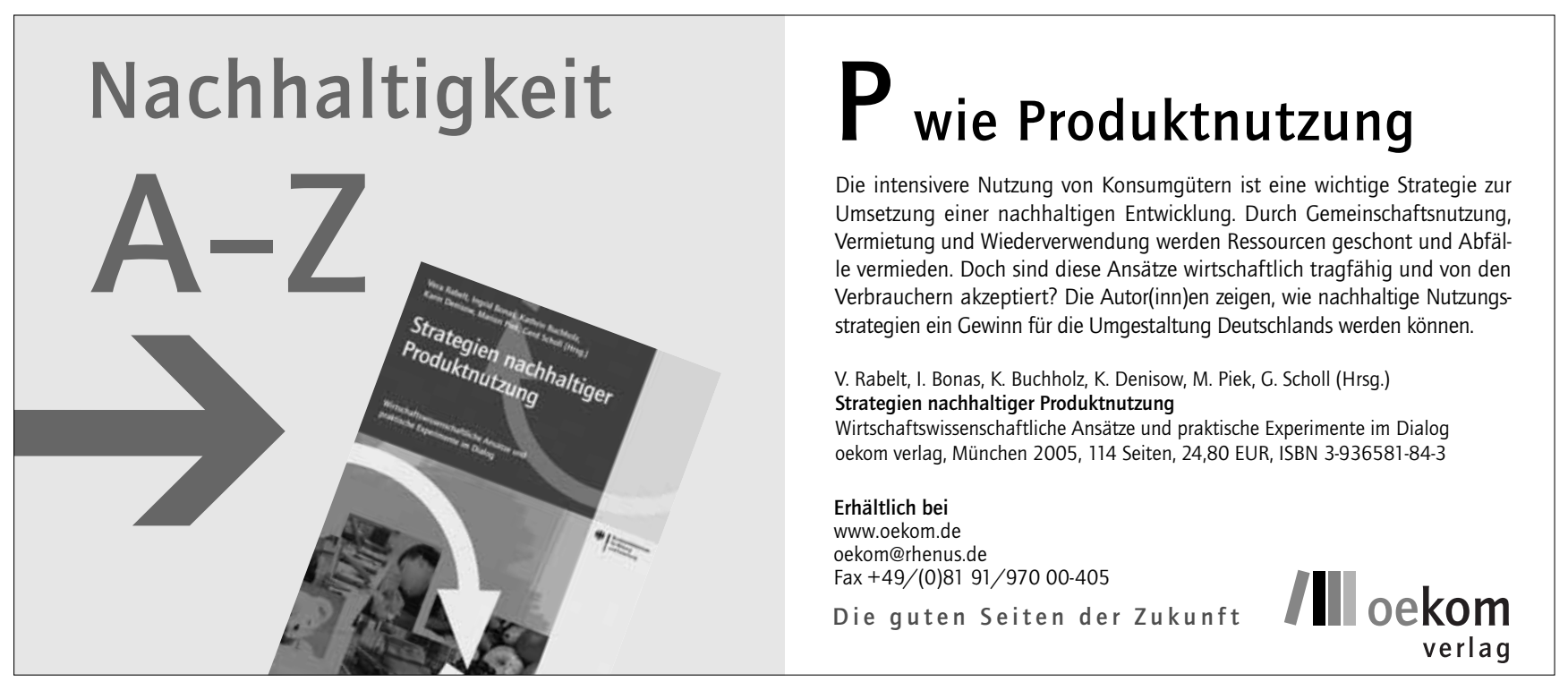


ge. Derartige Speicher stellen derzeit die klassische und am häufigsten eingesetzte Variante dar. Bivalente Speicher, also Speicher mit zwei Wärmetauschern, werden in Verbindung mit regenerativen Energieträgern bei Nachheizung durch einen Gas-, Öl- oder Pelletbrenner eingesetzt. Bivalente Speicher gewinnen derzeit durch den zunehmenden Einsatz regenerativer Energieträger, insbesondere der Solarthermie, deutlich an Relevanz. So wurden nach Angaben des Bundesverbandes Solarwirtschaft in den vergangenen fünf Jahren jährlich durchschnittlich etwa 90.000 Solarwärmeanlagen und die entsprechende Zahl an Speichern installiert.

\section{Wärmeverluste als zentrales Bewertungskriterium}

Warmwasserspeicher haben die zentrale Aufgabe, die Speicherung von Wärme über einen möglichst langen Zeitraum bei geringstmöglichen Wärmeverlusten zu gewährleisten. Der Wärmeverlust eines Speichers stellt demnach das zentrale Kriterium für die Vergabe des Umweltzeichens Blauer Engel dar. Es haben sich zur Bestimmung und Angabe des Wärmeverlustes zwei Verfahren und Messgrößen etabliert. Der Vorteil der Wärmeverlustrate (WVR) gegenüber dem Bereitschaftswärmeverlust (BWV) ist, dass es sich um eine europaweit verwendete Größe handelt, wohingegen der BWV eine deutsche Norm darstellt. Die WVR wird derzeit schwerpunktmäßig zur Bestimmung der Wärmeverluste von Solarspeichern herangezogen, jedoch ist eine Bestimmung der Wärmeverluste nach diesem Verfahren auch für alle anderen Speicher möglich.

Aufgrund der in der Regel größeren Speicheroberfläche weisen Speicher mit höheren Volumengrößen tendenziell höhere Wärmeverlustraten auf. Angaben zur WVR und/oder zum BWV sind in der Regel im technischen Merkblatt des Speichers zu finden. Eine Stichprobe der auf dem deutschen Markt verfügbaren Speicher in Bezug auf ihre jeweiligen Wärmeverluste offenbarte zum Teil deutliche Unterschiede. Dies kann anhand einer beispielhaften Berechnung des Energiebedarfs eines Ein- bis Zweifamilienhau- ses für die Trinkwasserbereitstellung verdeutlicht werden. Bei Nutzung eines Trinkwasserspeichers mit einem Volumen von 160 Litern, Speicher dieser Größe decken gegenwärtig etwa 70 Prozent des Marktes ab, beträgt der reine Wärmebedarf zur Trinkwassererwärmung ohne Wärmeverluste etwa $2.000 \mathrm{kWh}$ jährlich. Findet ein gut gedämmter Trinkwasserspeicher Verwendung, so ist in diesem Fall zusätzlich mit einem Verlust von jährlich etwa $400 \mathrm{kWh}$ zu rechnen, bei einem schlecht gedämmten Speicher beträgt der Verlust dagegen jährlich über $600 \mathrm{kWh}$.

Abb. 1: Energiebedarf für Trinkwassererwärmung.

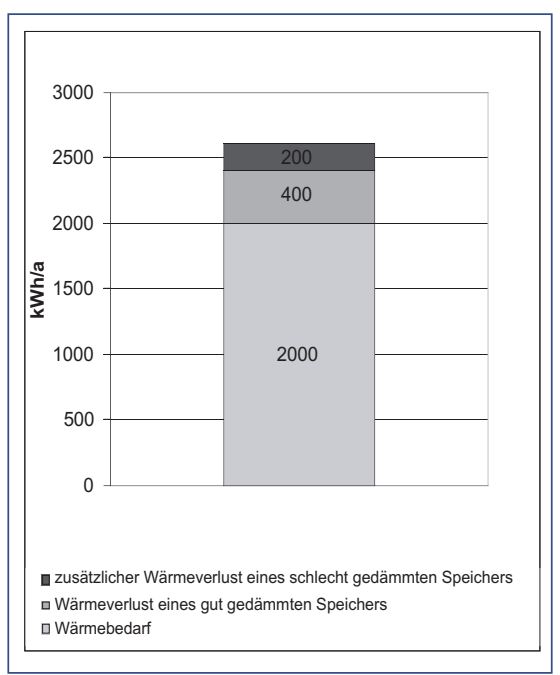

Quelle: eigene Darstellung

Der Wärmeverlust bei einem schlechten Speicher ist demzufolge um 50 Prozent höher als bei einem guten Speicher. Der Gesamtenergiebedarf zur Trinkwassererwärmung liegt damit bei einem schlechten Warmwasserspeicher um fast zehn Prozent über dem Bedarf bei Einsatz eines energieeffizienten Speichers. Mit Blick auf die übliche Lebensdauer der Speicher ergeben sich hier also erhebliche Effizienzunterschiede. Gleichzeitig sind die Verluste bei Puffer- und Kombispeichern aufgrund des in der Regel deutlich größeren Speichervolumens teilweise noch höher als bei den hier betrachteten Trinkwasserspeichern. Dadurch gehen gegebenenfalls erhebliche Anteile der solaren Energieerträge verloren. Die Auszeichnung hochwertiger Produkte durch ein Umweltzeichen Blauer Engel ist dement- sprechend mit einem hohen potenziellen Nutzen für den Verbraucher verbunden.

Neben der Wärmeverlustrate enthält die Vergabegrundlage weitere Vorgaben zu Qualität und Umweltverträglichkeit. Dies sind Hinweise zur recyclinggerechten Konstruktion der Geräte sowie zur Rücknahme und Verwertung der Speicher am Ende der Lebensdauer. Im Rahmen einer Fortschreibung der Vergabegrundlage wird darüber hinaus der Verzicht auf Flammschutzmittel bei den Dämmstoffen zu prüfen sein. Die entwickelten Vorgaben beabsichtigen, den an einer Kennzeichnung interessierten kleinen und mittelständischen Herstellern eine Zeichennahme bei begrenztem Aufwand zu ermöglichen.

\section{Umweltzeichen für Warm- wasserspeicher}

Das neue Umweltzeichen Blauer Engel für Warmwasserspeicher stellt eine wichtige Erweiterung bei der Verwendung des Blauen Engels für Komponenten des Wärmeerzeugungssystems dar. Ein Blauer Engel ermöglicht den Verbrauchern, auch bei der Auswahl ihres Warmwasserspeichers Gewissheit über die Einhaltung wesentlicher energetischer und ökologischer Kriterien zu erlangen. Gleichzeitig wird ein deutlicher Anreiz zur energetischen Verbesserung der Komponente Warmwasserspeicher gesetzt.

\section{Anmerkung}

(1) Das Institut für ökologische Wirtschaftsforschung (IÖW) hat im Auftrag des Umweltbundesamtes die Vergabegrundlage für Warmwasserspeicher entwickelt, vergeben durch Ökopol Hamburg im Rahmen des Clobalvorhabens.

I AUTOR + KONTAKT

Florian Wetzig ist Wissenschaftlicher Mitarbeiter im Bereich Nachhaltige Energiewirtschaft und Klimaschutz am Institut für ökologische Wirtschaftsforschung (IÖW). IÖW, Potsdamer Str. 105, 10785 Berlin. Tel. 030/884594-0, E-Mail: florian.wetzig@ioew.de 
(c) 20I0 Authors; licensee IÖW and oekom verlag. This is an article distributed under the terms of the Creative Commons Attribution Non-Commercial No Derivates License (http://creativecommons.org/licenses/by-nc-nd/3.o/), which permits unrestricted use, distribution, and reproduction in any medium, provided the original work is properly cited. 